\title{
Cerebral venous and sinus thrombosis
}

Thrombosis of the cerebral venous system or dural sinus thrombosis is a rare form of cerebrovascular disease that accounts for less than $1 \%$ of all strokes, with an associated mortality from 10 to $13 \% .{ }^{[1]}$ For many decades, cerebral venous thrombosis (CVT) was mainly linked to septic processes. However, the use of antibiotics became common and the infectious etiology of CVT was reduced. CVT is now typically recognized as a non septic disorder ${ }^{[2]} \mathrm{CVT}$ is characterized by the polymorphism of its neurological manifestations, difficulty in diagnosing it, the amount of different medical conditions that cause it, and its variable prognosis. ${ }^{[3]}$ The disorder can occur de novo as the first manifestation or can overlap on another existing clinical problem. In either case, it is always multifactorial and is variable in each patient. Each component of the Virchow's triad (endothelial damage, stasis, and hypercoagulability of blood) may in turn have several contributory factors to produce the final manifestation of CVT. These factors, which vary from patient to patient, operate together to produce cerebral cortical veins. Most often, only one of the etiological factors is prominent. It is essential to look for rarer and inherited causes of venous thrombosis. CVT can be the first manifestation of a thrombophilic state like polycythemia, thrombocytosis, leukocytosis, hyperhomocysteinemia, and antiphospholipid antibody syndrome. ${ }^{[4]}$ Usually, it has a favorable outcome, with mortality below 10\%. Magnetic resonance imaging (MRI) and magnetic resonance angiography are the best diagnostic methods and heparin is the first-line treatment. ${ }^{[2,4]}$

Two different mechanisms should be distinguished: Thrombosis of the cerebral veins, with local effects caused by venous obstruction, and thrombosis of the major sinuses, which causes intracranial hypertension.

\begin{tabular}{|l|l|}
\hline \multicolumn{2}{|c|}{ Access this article online } \\
\hline Quick Response Code: & Website: \\
\hline & www.ruralneuropractice.com \\
\cline { 2 - 3 } & \\
\hline & \\
\hline
\end{tabular}

In the majority of patients, these processes occur simultaneously. Clinical presentations of CVT are extremely variable as attested by the diversity of symptoms. Headache is the most frequent but least specific symptom; severe headache is present in more than $90 \%$ of adult patients. Usually, the headache mimics migraine but is persistently unilateral or diffuse and is not relieved after sleep, increases gradually over a couple of days, but can also start in a split second, mimicking intracerebral or subarachnoid hemorrhage. Focal syndrome (focal deficit, seizures, or both) mimicking and often mistaken for intracranial space-occupying lesion is not uncommon. Encephalopathy with multifocal signs, changes in mental status, stupor, or coma can occur suddenly over hours or days..$^{[3,4]}$

Computerized tomography allows detection of parenchymal lesions, which could have been caused by the thrombosis itself or by the rupture of dilated veins. The current gold standard is the combination of MRI to visualize the thrombosed vessel and magnetic resonance venography to detect the nonvisualization of the same vessel. The diagnostic yield of magnetic resonance venography does not differentiate between thrombosis and hypoplasia, a typical diagnostic dilemma for lateral sinuses. ${ }^{[5]}$ Brain parenchymal consequences are highly variable with T1- and T2-weighted images showing no abnormality in 30\% of the patients. In other cases, a localized or diffused brain swelling with edema, hemorrhage, or infarction is found. ${ }^{[4,6]}$

Quantitative measurement of a circulating D-dimer, a product of fibrin degradation, has been shown to be a very useful diagnostic tool in the managements of patients with suspected deep vein thrombosis and/or pulmonary embolism. Whether the D-dimer can play a similar role in the diagnostic approach to patients with suspected CVT remains controversial. ${ }^{[4]}$ In a statement of the American Heart Association and the American Stroke Association, the authors suggest that a normal D-dimer level maybe of help in identifying patients with a low probability of CVT, and that a normal D-dimer level should not preclude further evaluation if there is a strong clinical suspicion of CVT. ${ }^{[7]}$ 
Many disorders (medical, surgical, and gyneco-obstetric, genetic, and acquired prothrombotic disorders, cancer, hematological diseases, vasculitis, and other inflammatory systemic disorders, infection, brains tumors, arteriovenous malformations, head trauma, and infection of the ear, sinus, mouth, or neck) can cause CVT. The etiology is not identified in $15-20 \%$ of cases. Frequently, many risk factors are associated; hence, a systematic, complete etiological screening is necessary even if the cause is apparently obvious. ${ }^{[3]}$

Treatment of CVT combines causal treatment, antithrombotic treatment, and symptomatic treatment. The aims of antithrombotic treatment in CVT are to recanalize the occluded sinus or vein, to prevent the propagation of the thrombus, and to treat the underlying prothrombotic state..$^{[1,6,8]}$ Anticoagulants with body weight heparin or dose-adjusted intravenous heparin are widely used as first-line therapy on the basis of three randomized trials..$^{[1,6]}$ Endovascular thrombolysis can be applied at centers where the staff have experience in interventional radiology, and this treatment method should be restricted only to patients with poor prognosis. ${ }^{[9,10]}$ In patients who have symptoms of chronic intracranial hypertension, surgical drainage of the cerebrospinal fluid is indicated by a lumboperitoneal shunt. ${ }^{[10]}$

Prognosis of CVT has changed favorably over the last 30 years. In the past, it was considered a practically mortal illness. With MRI, mortality rate in the acute phase is $4,3 \%$ in evolution after 30 days. ${ }^{[1,9]}$
Malika Boucelma

Mohamed Lamine Debaghine Hospital, University of Algiers, Algeria

Address for correspondence: Mohamed Lamine Debaghine Hospital, University of Algiers, Algeria

E-mail: mboucelma@yahoo.fr

\section{References}

1. Ferro JM, Canhao P, Stam J, Bousser MG, Barinagarrementeria F; ISCVT Investigators. Prognosis of cerebral vein and dural sinus thrombosis: Results of the International Study on Cerebral Vein and Dural Sinus Thrombosis (ISCVT). Stroke 2004;35:664-70.

2. Stam J. Thrombosis of the cerebral veins and sinuses. N Engl J Med 2005;352:1791-8.

3. Bousser MG, Ross Russell RW. Cerebral venous thrombosis. Vol. 1. London: Saunders; 1992.

4. De Bruijn SF, Stam J, Kappelle LJ Thunderclap headache as first symptom of cerebral venous thrombosis. Lancet 1996;348:1623-5.

5. Horowitz M, Purdy P, Unwin H, Carstens G $3^{\text {rd }}$, Greenlee R, Hise J, et al. Treatment of dural sinus thrombosis using selective catheterization and urokinase. Ann Neurol 1995;38:58-67.

6. Bousser MG, Ferro JM. Cerebral venous thrombosis: An update. Lancet Neurol 2007;6:162-70.

7. Dentali F, Squizzato A, Marchesi E, Bonzini M, Ferro JM, Ageno WD. Dimer testing in the diagnosis of cerebral vein thrombosis: A systematic review and meta-analysis of the literature. J Thromb Haemost 2012;10:582-9.

8. Alvis-Miranda H, Castellar-Leones SM, Alcala-Cerra G, MoscoteSalazar LR. Cerebral sinus venous thrombosis: J Neurosci Rural Pract 2013;4:427-38.

9. Sasidharan PK. Cerebral vein thrombosis: Misdiagnosed and mismanaged. Thrombosis 2012; 2012:210676.

10. Einhäupl K, Bousser MG, de Bruijn SF, Ferro JM, Martinelli I, Masuhr F, et al. EFNS guidelines on the treatment of Cerebral venous and sinus thrombosis. Eur J Neurol 2006; 130:553-9.

How to cite this article: Boucelma M. Cerebral venous and sinus thrombosis. J Neurosci Rural Pract 2013;4:381-2.

Source of Support: Nil. Conflict of Interest: None declared.

Announcement

Android App

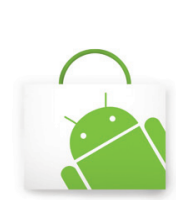

A free application to browse and search the journal's content is now available for Android based mobiles and devices. The application provides "Table of Contents" of the latest issues, which are stored on the device for future offline browsing. Internet connection is required to access the back issues and search facility. The application is compatible with all the versions of Android. The application can be downloaded from https://market.android.com/details?id=comm.app.medknow. For suggestions and comments do write back to us. 